\title{
DYNAMIC BEHAVIOUR COMPARISON OF THREE DIFFERENT MATHEMATICAL MODEL COMPleXities
}

\author{
Jiri Zatopek \& Zdenek Urednicek
}
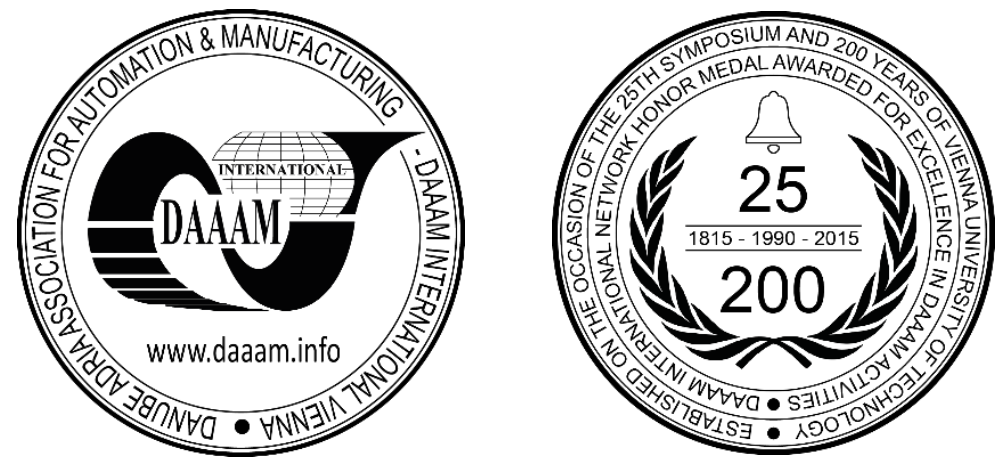

This Publication has to be referred as: Zatopek, J[iri] \& Urednicek, Z[denek] (2017). Dynamic Behaviour Comparison of Three Different Mathematical Model Complexities, Proceedings of the 28th DAAAM International Symposium, pp.0685-0693, B. Katalinic (Ed.), Published by DAAAM International, ISBN 978-3-902734-11-2, ISSN 1726-9679, Vienna, Austria

DOI: 10.2507/28th.daaam.proceedings.096

\begin{abstract}
Dynamic behaviour comparison of three different mathematical descriptions complexity for first 3 joints of 6 degrees of freedom robotic structure is presented in this article. Firstly, 3D CAD model is designed in SolidWorks, which is used as the basis for a physical and mathematical model. The CAD model is exported directly from SolidWorks to SimMechanics as a physical model which is considered as the most accurate replacement for a real model in this work. The first type of a mathematical model is the most precise but also the most complex; it is based on SolidWorks inertia matrices and matrix form of Lagrange's motion equations of the second kind. The second type of a mathematical model is created by each part replacement with a suitable simplified shape; classical integration approach with Lagrange's motion equations of the second kind is used. The third type of a mathematical model is based on the same approach as the second type, but all the objects are replaced by mass points. At the end, all the results of dynamic behaviour are compared with the physical model, for utilization in controller design.
\end{abstract}

Keywords: Model-Based-Design; Mathematical model; Simulation; Motion equation; SimMechanics

\section{Introduction}

Nowadays, a wide range of different support software tools are available; it provides many possibilities and ways to determine mathematical or physical model $[4,5,6,7,9,10,11,13,14,15,17,18,19,20]$. Accurate detection of a mathematical model is complicated in the case of a complex mechanical structure with many parts, and it should not be used for simulation purposes and controller determination at the same time. The chosen way is to split the problem between 2 models is: the most accurate model for a simulation as a real (physics) model substitution - physical model [4, 9, 14, 18] and appropriately simplified model purely for a controller determination - mathematical model [7, 19, 20].

For a mathematical and physical model, a CAD model is very helpful. SolidWorks is used for a CAD model design $[12,14,15,16,17,18]$. Only the shape and the material properties of an object are needed to know for a full dynamic description; these parameters are included in the CAD model. This means that the CAD model should always be the first step in an accurate dynamic simulation of any system. 
The physical model is exported directly from SolidWorks to the SimMechanics Second Generation [14, 15]. It includes all dimensions, joins and material properties of the CAD model. It is the most accurate model that includes all important shapes (inertia matrices) and mass details for dynamic simulation purposes [16, 17].

The first type of mathematical model is the most accurate but also the most complex. It is based on inertia matrices $[4,11]$, transformation matrices $[1,3,14,17,20]$, centres of mass position vectors $[2,16]$ and model masses. All of these parameters are taken from the SolidWorks CAD model. In this case, the matrix form of Lagrange's motion equations of the second kind is used $[2,16]$.

The second type of mathematical model uses shape simplification. Every link is replaced by a mass rod, every actuator is superseded by a mass point and significant cylindrical part is replaced by a mass cylindrical plate. The whole assembly is divided into many essential parts whose contribution of energy sumis the energy of the entire assembly.

The third type of mathematical model is similar to the second type, but all stationary objects are replaced by mass points. This mathematical model is the simplest, but its accuracy is the lowest.

In the results of this work, the differences between the above mentioned mathematical descriptions during dynamic simulation should be visible. These results should help to understand when it is better to use a more precise mathematical model and when the simplest model is sufficient as a basis for design control law.

\section{CAD model}

The SolidWorks CAD model consists of 9 parts as shown in Fig.1. Each part has an associated local coordinate system, the material and joins each other by structural bonds to the final fully bonded assembly $[12,14,15,16]$.

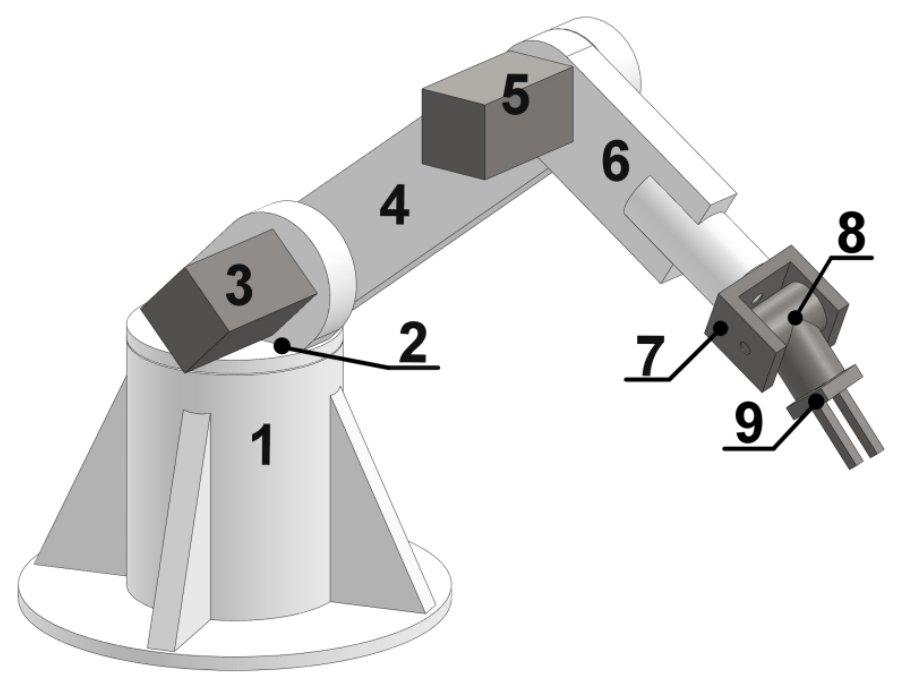

Fig. 1. The CAD model of the analysed system

The CAD model has 6 degrees of freedom (DOF) [3, 18] generally, but the first 3 joints have the most interesting dynamic behaviour and will be studied in this paper. Therefore, the last 3 DOF (parts 7, 8 and 9 in Fig. 1.) will be fixedly connected to each other and with the link 6.

\section{Physical model}

The SolidWorks model is exported directly into the SimMechanics Second Generation diagram along with all the linkages, dimensions and material properties $[14,15]$.

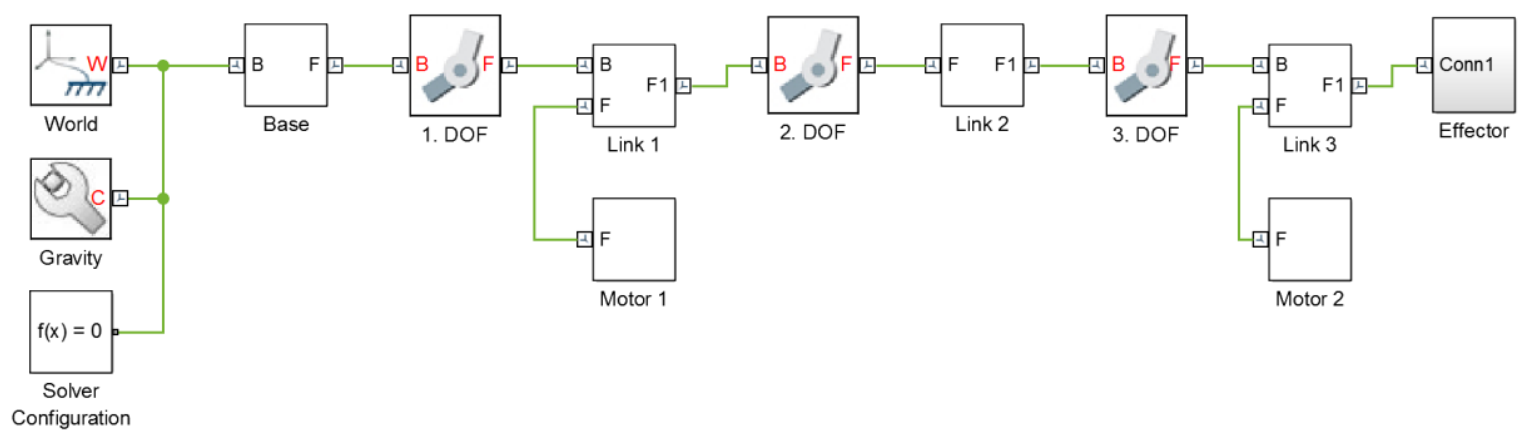

Fig. 2. SimMechanics Second Generation physical model 
A physical model is considered to be the most accurate substitute for a real physics system in case of precise CAD model which is the SimMechanics model based on. All mathematical models dynamic behaviours are compared with the physical model to see how accurate the mathematical models are.

\section{Mathematical models}

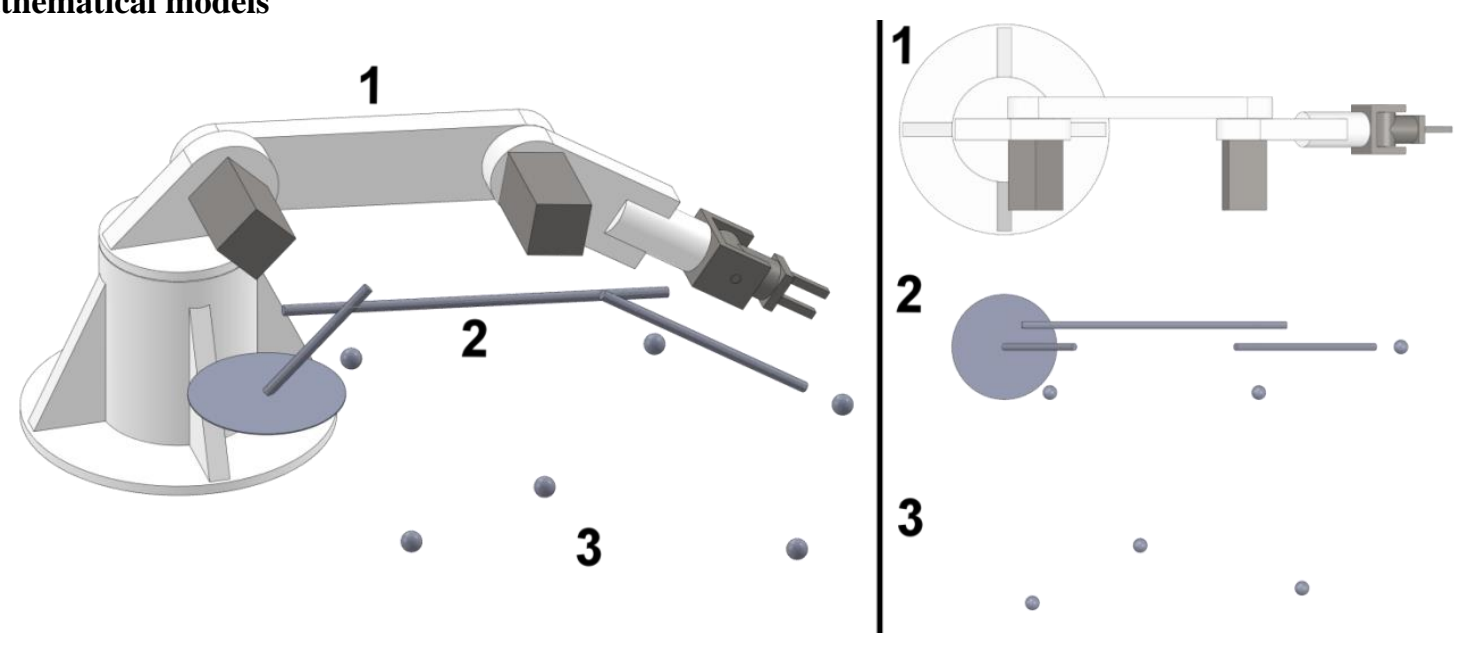

Fig. 3. Graphic preview of mathematical model simplifications

Three different CAD model simplifications are shown in Fig. 3. and their mathematical descriptions are derived below.

\subsection{Transformation of coordinate systems}

The transformation matrices are used to determine the position and orientation of a local coordinate system firmly connected with the component in the overall global coordinate system $[8,15]$. They are an integral part of any complex mathematical model determination.
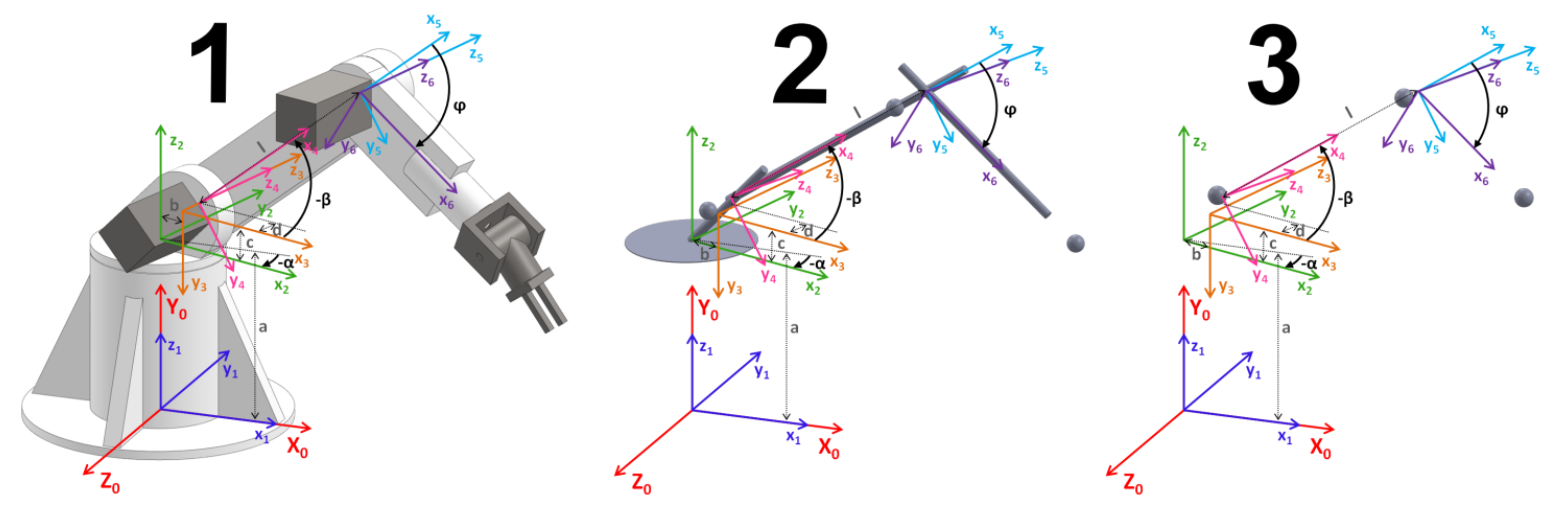

Fig. 4. Simplified models with the selected coordinate system placement

$$
\begin{aligned}
T_{1}={ }^{0} T_{2} & =\left[\begin{array}{cccc}
\cos (\alpha) & -\sin (\alpha) & 0 & 0 \\
0 & 0 & 1 & a \\
-\sin (\alpha) & -\cos (\alpha) & 0 & 0 \\
0 & 0 & 0 & 1
\end{array}\right] \\
T_{2}={ }^{o} T_{4}= & {\left[\begin{array}{cccc}
\cos (\beta) \cos (\alpha) & \cos (\alpha) \sin (\beta) & -\sin (\alpha) & b \cdot \cos (\alpha)-d \cdot \sin (\alpha) \\
\sin (\beta) & -\cos (\beta) & 0 & a+c \\
-\cos (\beta) \sin (\alpha) & -\sin (\alpha) \sin (\beta) & -\cos (\alpha) & -d \cdot \cos (\alpha)-b \cdot \sin (\alpha) \\
0 & 0 & 0 & 1
\end{array}\right] } \\
T_{3}={ }^{0} T_{6}= & {\left[\begin{array}{cccc}
\cos (\beta+\varphi) \cos (\alpha) & \cos (\alpha) \sin (\beta+\varphi) & -\sin (\alpha) & b \cdot \cos (\alpha)-d \cdot \sin (\alpha)+l \cdot \cos (\alpha) \cos (\beta) \\
\sin (\beta+\varphi) & -\cos (\beta+\varphi) & 0 & a+c+l \cdot \sin (\beta) \\
-\cos (\beta+\varphi) \sin (\alpha) & -\sin (\alpha) \sin (\beta+\varphi) & -\cos (\alpha) & -d \cdot \cos (\alpha)-b \cdot \sin (\alpha)-l \cdot \sin (\alpha) \cos (\beta) \\
0 & 0 & 0 & 1
\end{array}\right] }
\end{aligned}
$$


The centres of mass position vectors for the above mentioned local coordinate systems are different for each mathematical model simplification. In general, they are listed as:

$$
r_{1}={ }^{2} r_{2}=\left[\begin{array}{llll}
r_{11} & r_{12} & r_{13} & 1
\end{array}\right]^{T} ; \quad r_{2}={ }^{4} r_{4}=\left[\begin{array}{llll}
r_{21} & r_{22} & r_{23} & 1
\end{array}\right]^{T} \quad r_{3}={ }^{6} r_{6}=\left[\begin{array}{llll}
r_{31} & r_{32} & r_{33} & 1
\end{array}\right]^{T}
$$

For the next steps, the real model parameters are:

$$
\begin{array}{llll}
a=0,171 \mathrm{~m} ; & b=0,065 \mathrm{~m} ; & c=0,065 \mathrm{~m} ; & d=0,031 \mathrm{~m} ; \mathrm{l}=0,3 \mathrm{~m} \\
m_{1}=2,938 \mathrm{~kg} ; & m_{2}=1,008 \mathrm{~kg} ; & m_{3}=4,565 \mathrm{~kg} ; & \mathrm{g}=9,81 \mathrm{~m} \cdot \mathrm{s}^{-2}
\end{array}
$$

\subsection{The first mathematical model - no simplification}

The first model (Fig. 4. part 1) has a different approach to the motion equation derivation than the other two models. It uses the matrix form of Lagrange's motion equations of the second kind. This mathematical description is the most accurate of the three listed models because of inertia matrix using with a centre of mass position vectors from the CAD model, which are with transformation matrices all necessary parameters to need to know.

Motion equations matrix form has the general form $[2,16]$ :

$$
\mathrm{D}(\mathrm{q}) \cdot \ddot{\mathrm{q}}+\mathrm{H}(\mathrm{q}, \dot{\mathrm{q}})+\mathrm{G}(\mathrm{q})=\mathrm{T} \quad \Rightarrow \quad \mathrm{D}(\alpha, \beta, \varphi) \cdot\left[\begin{array}{c}
\ddot{\alpha} \\
\ddot{\beta} \\
\ddot{\varphi}
\end{array}\right]+\mathrm{H}(\alpha, \dot{\alpha}, \beta, \dot{\beta}, \varphi, \dot{\varphi})+\mathrm{G}(\alpha, \beta, \varphi)=\left[\begin{array}{c}
\mathrm{T}_{1} \\
\mathrm{~T}_{2} \\
\mathrm{~T}_{3}
\end{array}\right]
$$

in which:

$$
\begin{aligned}
& D_{i, j}=\sum_{r=\max \{i, j\}}^{n} \operatorname{tr}\left[\frac{\partial^{0} T_{r}}{\partial q_{j}} \cdot{ }^{r} \bar{I}_{r}\left(\frac{\partial^{0} T_{r}}{\partial q_{i}}\right)^{T}\right] \\
& H_{i}=\sum_{k=1}^{n} \sum_{m=1}^{n} H_{i k m} \dot{q}_{k} \dot{q}_{m} \\
& H_{i k m}=\sum_{r=m a x\{i, k, m\}}^{n} \operatorname{tr}\left[\frac{\partial^{2^{0}} T_{r}}{\partial q_{k} \partial q_{m}} \cdot{ }^{r} \bar{I}_{r}\left(\frac{\partial^{0} T_{r}}{\partial q_{i}}\right)^{T}\right] \\
& G_{i}=-\sum_{r=i}^{n} m_{r}\left({ }^{o} \bar{g}\right)^{T} \cdot \frac{\partial^{0} T_{r}}{\partial q_{i}} \cdot{ }^{r} \bar{r}_{r}
\end{aligned}
$$

As could be seen, the only two other variables need to be known. These parameters are found in the SolidWorks CAD model.

The centres of mass position vectors are:

$$
r_{1}=\left[\begin{array}{llll}
0,057 & -0,053 & 0,058 & 1
\end{array}\right]^{T} ; \quad r_{2}=\left[\begin{array}{llll}
-0,15 & 0 & -0,004 & 1
\end{array}\right]^{T} ; \quad r_{3}=\left[\begin{array}{llll}
0,099 & 0 & -0,065 & 1
\end{array}\right]^{T}
$$

The pseudo-inertia matrices are:

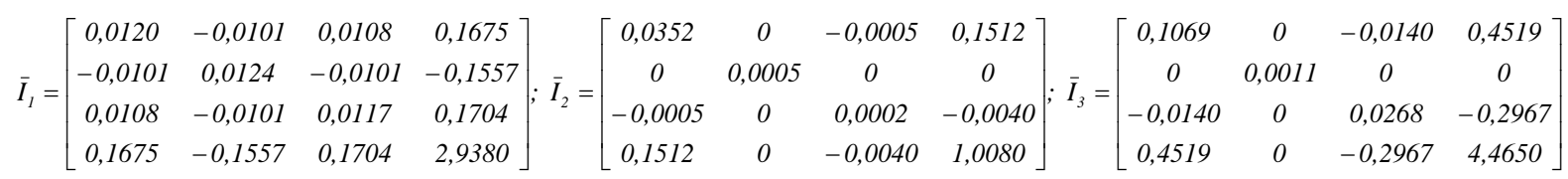

The specific solution for the system shown in equation (6) is:

$$
\begin{aligned}
& \mathbf{D 1}=\left[\begin{array}{ccc}
D_{11} & 5,008 \cdot 10^{-5} \sin (\beta+\varphi)-0,04242 \sin (\beta) & 5,008 \cdot 10^{-5} \sin (\beta+\varphi) \\
5,008 \cdot 10^{-5} \sin (\beta+\varphi)-0,04242 \sin (\beta) & 0,2712 \cos (\varphi)+0,5546 & 0,1356 \cos (\varphi)+0,108 \\
5,008 \cdot 10^{-5} \sin (\beta+\varphi) & 0,1356 \cos (\varphi)+0,108 & 0,108
\end{array}\right] \\
& D_{11}=0,1356 \cos (2 \beta+\varphi)+0,2228 \cos (2 \beta)+0,05875 \cos (2 \beta)+0,1977 \cos (\beta)+0,1356 \cos (\varphi)+0,05292 \cos (2 \beta+2 \varphi)+0,3389
\end{aligned}
$$


$\mathbf{H 1}=\left[\begin{array}{c}\dot{\beta}^{2}\left[5,008 \cdot 10^{-5} \cos (\beta+\varphi)-0,04242 \cos (\beta)\right]+1,002 \cdot 10^{-3} \cos (\beta+\varphi) \dot{\beta} \dot{\varphi}-2 \dot{\alpha} \dot{\beta}[0,1356 \sin (2 \beta+\varphi)+0,2228 \sin (2 \beta)+ \\ +0,02938 \sin (\beta+\varphi)+0,09885 \sin (\beta)+0,05292 \sin (2 \beta+2 \varphi)]+5,008 \cdot 10^{-5} \cos (\beta+\varphi) \dot{\varphi}-2 \dot{\alpha} \dot{\varphi}[0,06779 \sin (2 \beta+\varphi)+ \\ +0,02938 \sin (\beta+\varphi)+0,06779 \sin (\varphi)+0,05292 \sin (2 \beta+2 \varphi)] \\ \dot{\alpha}^{2}[0,05292 \sin (2 \beta+2 \varphi)+0,1356 \sin (2 \beta+\varphi)+0,2228 \sin (2 \beta)+0,02938 \sin (\beta+\varphi)+0,09885 \sin (\beta)]- \\ -0,1356 \sin (\varphi) \dot{\varphi}^{2}-0,2712 \sin (\varphi) \dot{\beta} \dot{\varphi} \\ \dot{\alpha}^{2}[0,06779 \sin (2 \beta+2 \varphi)+0,02938 \sin (\beta+\varphi)+0,06779 \sin (\varphi)+0,05292 \sin (2 \beta+2 \varphi)]+0,1356 \sin (\varphi) \dot{\beta}^{2}\end{array}\right]$

$\mathbf{G 1}=\left[\begin{array}{c}0 \\ 4,433 \cos (\beta+\varphi)+14,92 \cos (\beta) \\ 4,433 \cos (\beta+\varphi)\end{array}\right]$

\subsection{The second mathematical model - shape simplification}

The second model (Fig. 4. part 2) uses shape simplification as described in the introduction part. The Lagrange equations of the second kind were used to find the motion equations. In order to use Lagrange equations, it is necessary to define the kinetic and potential energy of the whole system. The transformation matrices and the centre of mass position vectors are used to determine the absolute position and velocity of each simplified part. This mathematical description is not as accurate as the previous model but should be sufficient even for simulation purposes because of the mass distribution cause - it is analyzed in results comparison.

Due to the motion equations derivation complexity (each part has similar but not the same approach to find kinetic and potential energy) will be described only one simplified part (Fig. 1. part 4) kinetic/potential energy determination and then the motion equations will be derived.

The position vector in the global coordinate system:

$$
p_{1}=\mathbf{T}_{2} \cdot r_{2}=\left[\begin{array}{llll}
p_{11} & p_{12} & p_{13} & 1
\end{array}\right]^{T}
$$

The velocity vector in the global coordinate system:

$$
v_{1}=\frac{d p_{1}}{d t}=\left[\begin{array}{llll}
v_{11} & v_{12} & v_{13} & 1
\end{array}\right]^{T}
$$

The kinetic energy:

$$
\begin{aligned}
& d E_{K 1}=\frac{1}{2} \cdot d m_{1} \cdot\left(v_{11}{ }^{2}+v_{12}{ }^{2}+v_{13}{ }^{2}\right) \\
& E_{K 1}=\iint_{D} d E_{K 1}
\end{aligned}
$$

The potential energy (gravitation is in the global axis $\mathrm{Y}$ direction, therefore the $p_{12}$ position is used):

$$
E_{P 1}=m_{1} \cdot g \cdot p_{12}
$$

The kinetic and potential energy of the other six parts (Fig. 1.) is determined in a similar way like for the abovementioned part. The total kinetic and potential energy of the system is:

$$
E_{K}=\sum_{i=1}^{7} E_{K i} \quad E_{P}=\sum_{i=1}^{7} E_{P i}
$$

The Lagrangian:

$$
L=E_{K}-E_{P}
$$

The final motion equations:

$$
\frac{d}{d t}\left(\frac{\partial L}{\dot{\alpha}}\right)-\frac{\partial L}{\alpha}=T_{1} ; \quad \frac{d}{d t}\left(\frac{\partial L}{\dot{\beta}}\right)-\frac{\partial L}{\beta}=T_{2} ; \quad \frac{d}{d t}\left(\frac{\partial L}{\dot{\varphi}}\right)-\frac{\partial L}{\varphi}=T_{3} ;
$$

Equation (19) can be also rewritten into the motion equations matrix form shown in equation (6). For the parameters in equation (5) and the corresponding centres of mass position vectors, the motion equations for the second mathematical model are: 
$\mathbf{D 2}=\left[\begin{array}{ccc}D_{11} & -0,04211 \sin (\beta) & 0 \\ -0,04211 \sin (\beta) & 0,2666 \cos (\varphi)+0,5497 & 0,1333 \cos (\varphi)+0,1045 \\ 0 & 0,1333 \cos (\varphi)+0,1045 & 0,1045\end{array}\right]$

$D_{11}=0,1333 \cos (2 \beta+\varphi)+0,2226 \cos (2 \beta)+0,05776 \cos (\beta+\varphi)+0,1975 \cos (\beta)+0,1333 \cos (\varphi)+0,05227 \cos (2 \beta+2 \varphi)+0,3314$

$\mathbf{H 2}=\left[\begin{array}{l}-\dot{\alpha} \dot{\varphi}[0,1333 \sin (2 \beta+\varphi)+0,05776 \sin (\beta+\varphi)+0,1333 \sin (\varphi)+0,1045 \sin (2 \beta+2 \varphi)]-0,04211 \cos (\beta) \dot{\beta}^{2}- \\ -\dot{\alpha} \dot{\beta}[0,2666 \sin (2 \beta+\varphi)+0,4451 \sin (2 \beta)+0,05776 \sin (\beta+\varphi)+0,1975 \sin (\beta)+0,1045 \sin (2 \beta+2 \varphi)] \\ \dot{\alpha}^{2}[0,05227 \sin (2 \beta+2 \varphi)+0,1333 \sin (2 \beta+\varphi)+0,2226 \sin (2 \beta)+0,02888 \sin (\beta+\varphi)+0,09873 \sin (\beta)]- \\ -\dot{\varphi}[0,2666 \sin (\varphi) \dot{\beta}+0,1333 \sin (\varphi) \dot{\varphi}] \\ \dot{\alpha}^{2}[0,05227 \sin (2 \beta+2 \varphi)+0,06665 \sin (2 \beta+\varphi)+0,02888 \sin (\beta+\varphi)+0,06665 \sin (\varphi)]+0,1333 \sin (\varphi) \dot{\beta}^{2}\end{array}\right]$

$\mathbf{G 2}=\left[\begin{array}{c}0 \\ 4,359 \cos (\beta+\varphi)+14,9 \cos (\beta) \\ 4,359 \cos (\beta+\varphi)\end{array}\right]$

This mathematical description is not too complicated compared to the previous equations (10), (11) and (12). Nevertheless, there is one more solution with a simpler mathematical description determination.

\subsection{The third mathematical model - mass point simplification}

The third model (Fig. 4. part 3) uses mass point simplification which quantity is equal to the DOF number. The Lagrange equations of the second kind were also used to the motion equations determination. There is the same approach as in the previous chapter 4.3 , but all the fixed parts are replaced by the mass points located in their centre of mass. Their number is three because the model has three DOF. This mathematical description is not as accurate as previous two models and should not be used for the simulation purposes because all the mass is concentrated in the centre of mass of each fixed body parts only. This means the mass distribution has been disturbed.

However, this model is easiest to derive and should be the most appropriate for the design control law purposes because of its expected simplicity. The differences between dynamic behaviour will be determined in the next chapter. The third model's motion equations matrix form for the parameters in equation (5) and appropriate centres of mass position vectors are:

$$
\mathbf{D 3}=\left[\begin{array}{ccc}
D_{11} & -0,01537 \sin (\beta+\varphi)-0,04248 \sin (\beta) & -0,01537 \sin (\beta+\varphi) \\
-0,01537 \sin (\beta+\varphi)-0,04248 \sin (\beta) & 0,2712 \cos (\varphi)+0,4783 & 0,1356 \cos (\varphi)+0,04474 \\
-0,01537 \sin (\beta+\varphi)+0,04474 & 0,1356 \cos (\varphi)+0,04474 & 0,04474
\end{array}\right]
$$

$D_{11}=0,1356 \cos (2 \beta+\varphi)+0,2168 \cos (2 \beta)+0,05875 \cos (\beta+\varphi)+0,1977 \cos (\beta)+0,1356 \cos (\varphi)+0,02237 \cos (2 \beta+2 \varphi)+0,2865$

$$
\mathbf{H 3}=\left[\begin{array}{l}
-\dot{\alpha} \dot{\varphi}[0,1356 \sin (2 \beta+\varphi)+0,05875 \sin (\beta+\varphi)+0,1356 \sin (\varphi)+0,04474 \sin (2 \beta+2 \varphi)]-0,04248 \cos (\beta) \dot{\beta}^{2}- \\
-\dot{\alpha} \dot{\beta}[0,2712 \sin (2 \beta+\varphi)+0,4335 \sin (2 \beta)+0,05875 \sin (\beta+\varphi)+0,1977 \sin (\beta)+0,04474 \sin (2 \beta+2 \varphi)]- \\
-0,01537 \cos (\beta+\varphi) \dot{\beta}^{2}-0,01537 \cos (\beta+\varphi) \dot{\varphi}^{2}-0,03073 \cos (\beta+\varphi) \dot{\beta} \dot{\varphi} \\
\dot{\alpha}^{2}[0,02237 \sin (2 \beta+2 \varphi)+0,1356 \sin (2 \beta+\varphi)+0,2168 \sin (2 \beta)+0,02938 \sin (\beta+\varphi)+0,09885 \sin (\beta)]- \\
-\dot{\varphi}[0,2712 \sin (\varphi) \dot{\beta}+0,1356 \sin (\varphi) \dot{\varphi}] \\
\dot{\alpha}^{2}[0,02237 \sin (2 \beta+2 \varphi)+0,06779 \sin (2 \beta+\varphi)+0,02938 \sin (\beta+\varphi)+0,06779 \sin (\varphi)]+0,1356 \sin (\varphi) \dot{\beta}^{2}
\end{array}\right]
$$

$$
\mathbf{G 3}=\left[\begin{array}{c}
0 \\
4,433 \cos (\beta+\varphi)+14,92 \cos (\beta) \\
4,433 \cos (\beta+\varphi)
\end{array}\right]
$$

As could be seen, the simplest model with only three mass points does not mean simplest final motion equations. The second mathematical model is more accurate and not too complex, but its determination was hardest. 


\section{Comparison of results}

The resulting motion equations are compared with the physical model torque outputs in Fig. 5. The SimMechanics physical model is considered to be the most accurate dynamic model here and therefore, any deviation indicates an inaccurate approximation.
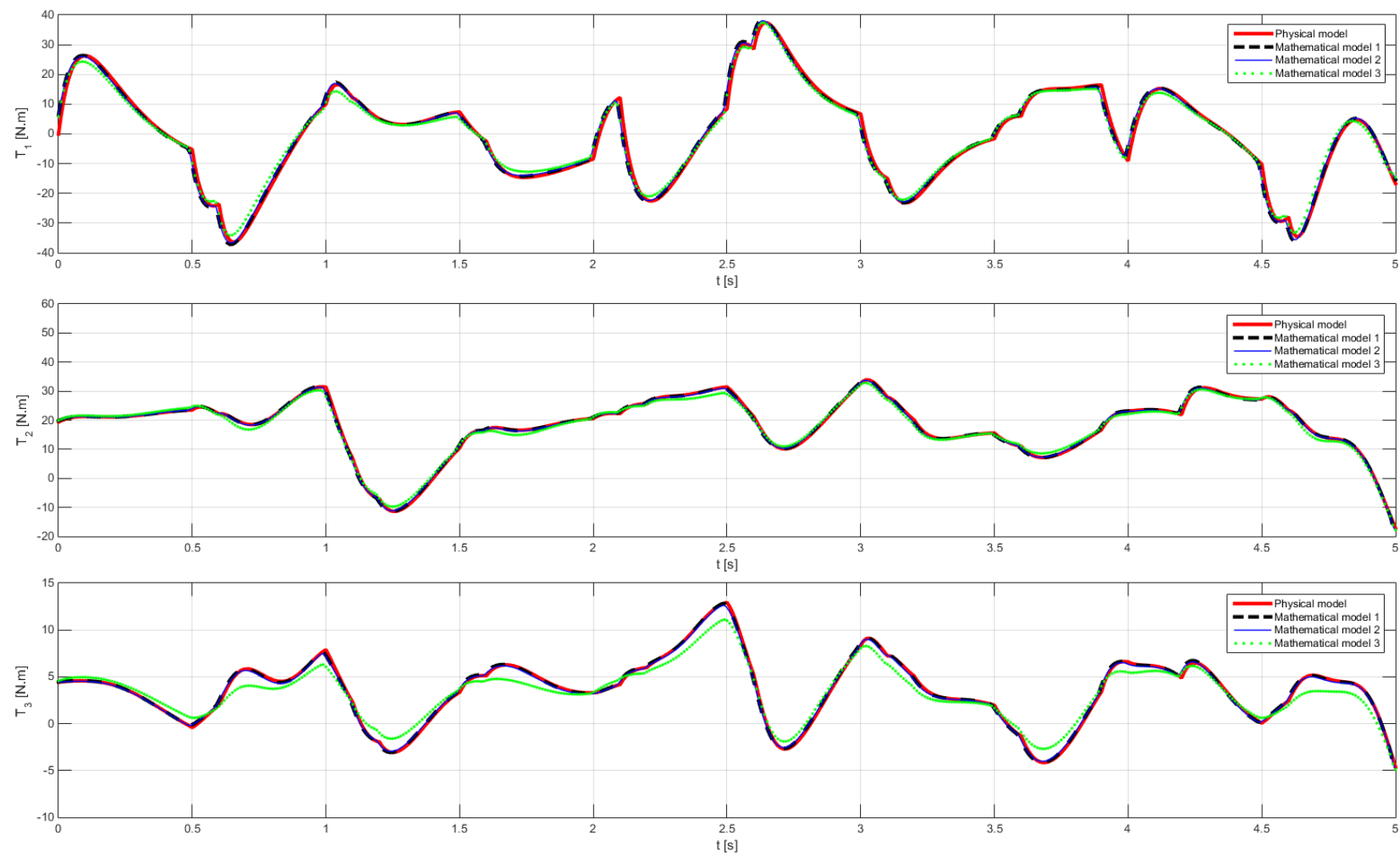

Fig. 5. The physical and the three different mathematical models comparison

As shown in Fig. 5., all the model's dynamic behaviour is very similar, only in the third DOF third mathematical model output is more obvious deflection. The motion, which the listed torques correspond to, was chosen as the most dynamic technological movement as could be required. For more detailed research, the Fig. 5. is enlarged in Fig. 6.
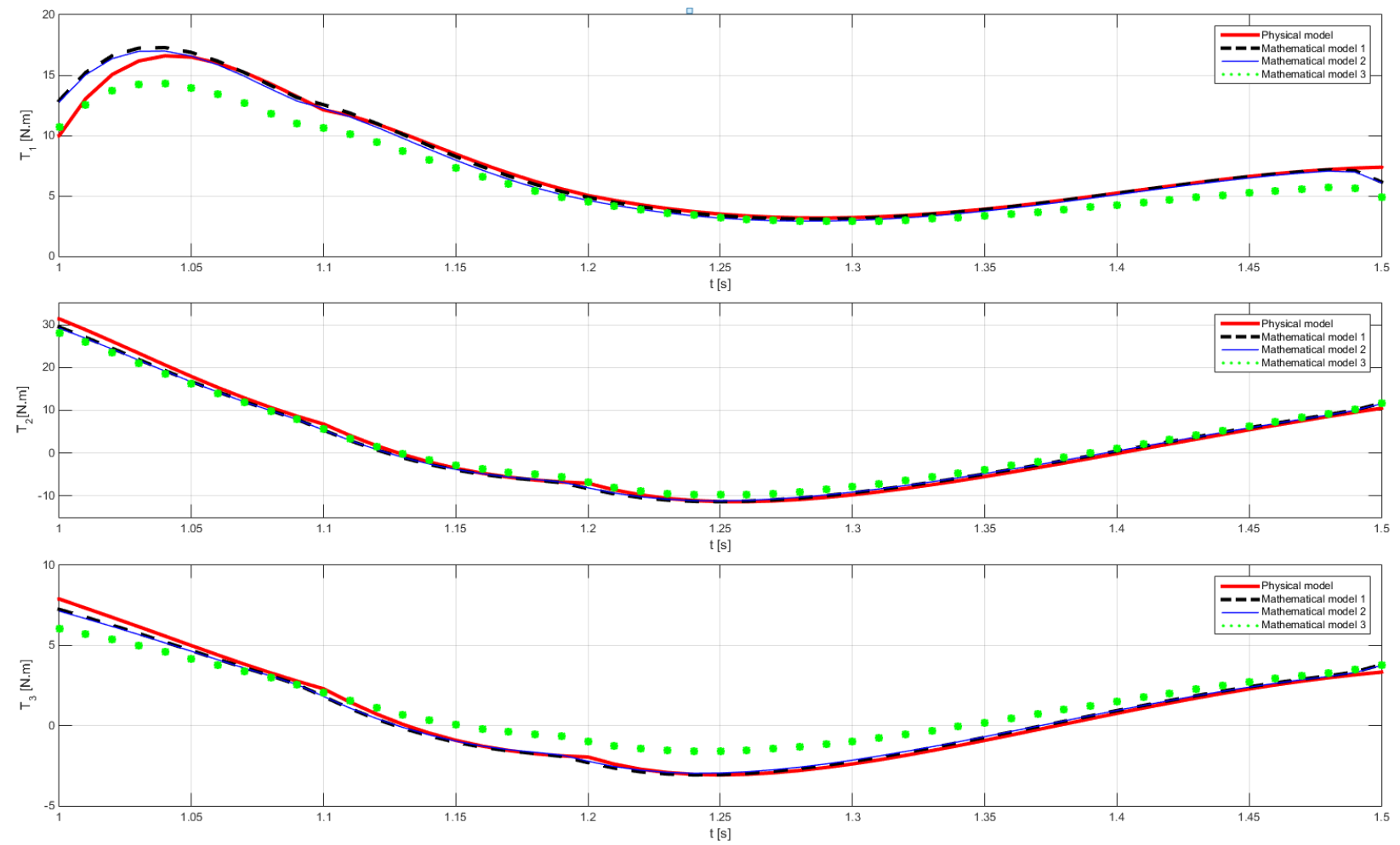

Fig. 6. The physical and the three different mathematical models comparison - detailed view 
The differences between all the mathematical models and the physical model are clear now. The red thick solid line shows the physical model. The black dashed line associated with the first and the most accurate mathematical model almost overlaps the thin blue line related to the second model (shape simplification). All three of the above-mentioned lines are highly similar and the dynamic behaviour for all of them is essentially the same. The last green dot line associated with the third mathematical model (mass point simplification) is also noticeably similar, but this model should not be used for a simulation purpose, thus the real model substitution, because of its dynamic deviation compares to the physical model. The dynamic deflection is not crucial, but the other two mathematical models are better for this purpose for certain.

\section{Conclusion}

The effort in this work was devoted to the dynamic behaviour comparison of three different mathematical models. As could be found, everything starts with a CAD model. It is an essential part of every accurate dynamic model determination. The CAD model was exported to the SimMechanics physical model which is considered to be the most accurate replacement of a real physics system. All the dynamic behaviours of the mathematical models have been compared with the physical model to see how accurate the mathematical models are.

The first mathematical model derivation uses matrices of inertia from CAD model. That means an accurate description of mass distribution directly in motion equations. This mathematical model is the most accurate, but a physical interpretation is not clear during a derivation. Also, calculation errors are very difficult to find.

The second mathematical model uses an integration approach with every part simplification. This approach is the most complicated in terms of derivation, but it is intuitive and accuracy can be continuously explored. Its accuracy is, surprisingly, almost as precise as the first mathematical model. It means that the shape of any part is not decisive in the case of an appropriate approximation selection.

The third mathematical model derivation is similar to the second type, but all stationary objects are replaced with mass points. The accuracy of a mass distribution description is lowest and the shape of motion equations is not as simple as expected. However, the own motion equations derivation is the simplest.

Ultimate dynamic behaviour is highly similar to the first and second mathematical model and based on their accuracy; both mathematical models can be even used as simulation models. The third mathematical model has also satisfactory accuracy which is more than enough for the control law design but should not be used as a real model substitution for simulation purposes.

All things considered, the shape precision directly in motion equations has not a significant influence in a dynamic behaviour description as could be expected if a centre of mass position is known. Further work could be focused on the use of derived motion equations to the design control law and comparison of on that basis designed regulators accuracy.

\section{References}

[1] Denavit, J. \& Hartenberg, R. S. (1955). ASME Journal of Applied Mechanics”, A kinematic notation for lower-pair mechanisms based on matrices, Vol. 77, No. 22, June 1955, pp. 215-221.

[2] R. N. Jazar. (2010). Theory of Applied Robotics, Springer US, ISBN: 978-1-4419-1750-8, Boston, MA

[3] Damic, V. \& Cohodar, M. (2016). Dynamic Analysis and Visualization of Spatial Manipulators with Closed Structure, Proceedings of the 26th DAAAM International Symposium, pp.0109-0117, B. Katalinic (Ed.), Published by DAAAM International, ISBN 978-3-902734-07-5, ISSN 1726-9679, Vienna, Austria, DOI: 10.2507/26th.daaam.proceedings.016

[4] Shala, A., Likaj, R., Bruqi, M. \& Bajrami, X. (2014). Propulsion Effect Analysis of 3Dof Robot under Gravity, Proceedings of the 25th DAAAM International Symposium, pp.206-212, B. Katalinic (Ed.), Published by Elsevier Ltd, ISSN 1877-7058, Vienna, Austria, DOI: 10.1016/j.proeng.2015.01.359

[5] Dragomirescu, C. G. \& Iliescu, V. (2010). About the Stability of the Motion of a Dynamic System in a Particular Case, Annals of DAAAM for 2010 \& Proceedings of the 21st International DAAAM Symposium, 20-23rd October 2010, Zadar, Croatia, ISSN 1726-9679, ISBN 978-3-901509-73-5, Katalinic, B. (Ed.), pp. 0197-0198, Published by DAAAM International Vienna, Vienna

[6] Copilusi, C. P.; Marin, M. I. \& Rusu, L. D. (2010). Design of a New Human Knee Prosthesis Based on CAM Mechanism, Annals of DAAAM for 2010 \& Proceedings of the 21st International DAAAM Symposium, 20-23rd October 2010, Zadar, Croatia, ISSN 1726-9679, ISBN 978-3-901509-73-5, Katalinic, B. (Ed.), pp. 0709-0710, Published by DAAAM International Vienna, Vienna

[7] Shala, A. \& Likaj, R. (2010). Fuzzy Logic Control and 3D Simulation of Road Vehicle, Annals of DAAAM for 2010 \& Proceedings of the 21st International DAAAM Symposium, 20-23rd October 2010, Zadar, Croatia, ISSN 1726-9679, ISBN 978-3-901509-73-5, Katalinic, B. (Ed.), pp. 1457-1458, Published by DAAAM International Vienna, Vienna

[8] Ciortan, M. (2011). Kinematic Analysis of Jaw Movements, Annals of DAAAM for 2011 \& Proceedings of the 22nd International DAAAM Symposium, 23-26th November 2011, Vienna, Austria, Volume 22, No. 1, ISSN 17269679, ISBN 978-3-901509-83-4, Katalinic, B. (Ed.), pp. 1599-1600, Published by DAAAM International Vienna, Vienna 
[9] Damic, V. \& Cohodar, M. (2014): Dynamic Analysis of Stewart Platform by Bond Graphs, 25th DAAAM International Symposium on Intelligent Manufacturing and Automation, 23-30th November 2014, Vienna, Austria, ISSN: 1877-7058, Procedia Engineering (2015), Volume 100, Katalinic, B. (Ed.), pp. 226 - 233, Published by DAAAM International Vienna, Vienna

[10] Vu, T. M.: Vehicle Steering Dynamic Calculation and Simulation, Annals of DAAAM for 2012 \& Proceedings of the 23rd International DAAAM Symposium, ISBN 978-3-901509-91-9, ISSN 2304-1382, pp 0237 - 0242 , Editor Branko Katalinic, Published by DAAAM International, Vienna, Austria, 2012

[11] Gebel, E. S. (2014): Dynamic Mathematical Modeling of Dynamics of Multi-Lever Linkages, 25th DAAAM International Symposium on Intelligent Manufacturing and Automation, 23-30th November 2014, Vienna, Austria, ISSN: 1877-7058, Procedia Engineering (2015), Volume 100, Katalinic, B. (Ed.), pp. 1562 - 1571, Published by DAAAM International Vienna, Vienna

[12] Aburaia, M.; Markl, E. \& Stuja, K. (2014): New Concept for Design and Control of 4 Axis Robot Using the Additive Manufacturing Technology, 25th DAAAM International Symposium on Intelligent Manufacturing and Automation, 23-30th November 2014, Vienna, Austria, ISSN: 1877-7058, Procedia Engineering (2015), Volume 100, Katalinic, B. (Ed.), pp. 1364 - 1369, Published by DAAAM International Vienna, Vienna

[13] Isaenko, S.; Sochivko, O.; Shardyko, I. \& Titov, V. (2016). Simulation of Manipulator as a Part of Space Robotic System Weightlessness Device, Proceedings of the 26th DAAAM International Symposium, pp.0554-0561, B. Katalinic (Ed.), Published by DAAAM International, ISBN 978-3-902734- 07-5, ISSN 1726-9679, Vienna, Austria DOI: $10.2507 / 26$ th.daaam.proceedings.076

[14] Damic, V.; Cohodar, M. \& Tvrtkovic, M. (2016). Inverse Dynamic Analysis of Hobby Robot uArm by Matlab/Simulink, Proceedings of the 27th DAAAM International Symposium, pp.0095-0101, B. Katalinic (Ed.), Published by DAAAM International, ISBN 978-3-902734-08-2, ISSN 1726-9679, Vienna, Austria DOI: 10.2507/27th.daaam.proceedings.014

[15] Zatopek, J. (2016). Simulation and Visualisation of a Laboratory Model by Support Software Tools Connection, Proceedings of the 27th DAAAM International Symposium, pp.0749-0755, B. Katalinic (Ed.), Published by DAAAM International, ISBN 978-3-902734-08-2, ISSN 1726-9679, Vienna, Austria DOI: $10.2507 / 27$ th.daaam.proceedings. 108

[16] Urednicek, Z. (2012). Robotika, T. Bata university in Zlin, ISBN: 978-80-7454-223-7, Zlin

[17] Gouasmi, M.; Ouali, M.; Fernini, B. \& Meghatria, M. (2012): Kinematic Modelling and Simulation of a 2-R Robot Using SolidWorks and Verification by MATLAB/Simulink, International Journal of Advanced Robotic Systems, pp. 245-257, Volume 9, Issue 6, DOI: 10.5772/50203

[18] Udai, A. D.; Rajeevlochana, CG \& Saha, S. K. (2011): Dynamic Simulation of a KUKA KR5 Industrial Robot using MATLAB SimMechanics, 15th National Conference on Machines and Mechanisms, pp. 1-8, 30th November to 2nd December 2011, Chennai, Tamilnadu, India, NaCoMM2011-96

[19] TLALE, N. S. \& ZHANG P. (2005): Teaching the Design of Parallel Manipulators and Their Controllers Implementing MATLAB, Simulink, SimMechanics and CAD*, Int. J. Engng Ed. Volume 21, No. 5, pp. 838-845, 0949-149X/91

[20] Aldoom, S.; Adam, A.; Ji-Pin, Z. \& Yi-hua, Z. (2017): Modeling and Simulation of 5DOF Robot Manipulator and Trajectory using Matlab and CATIA, 3rd International Conference on Control, Automation and Robotics, pp. 1-5, ISBN: 978-1-5090-6088-7, DOI: 10.1109/ICCAR.2017.7942657 